\title{
Study of the mechanical behavior of the optical fiber by a mark-tracking method
}

\author{
V. Chean, E. Robin, R. El Abdi and J-C. Sangleboeuf \\ LARMAUR Equipe Verres ERL CNRS 6274, PPT - Bâtiment 10B - Campus de Beaulieu, 35042 \\ Rennes Cedex, France
}

\begin{abstract}
The mark-tracking method was used in the uniaxial tensile test to determine the elastic properties of optical fibers. The mark-tracking method is based on the followup of two markers on the specimen with the help of an image processing technique. It allows us to determine the true strain with respect to the small strains assumption $(e \leq 1 \%)$ or the finite strains $(e>1 \%)$ without any impact of the rigid solid movement neither pulley fiber sliding on the measured strain. Optical fibers used in this study are commercial Verrillon single mode silica fibers, $125 \mu \mathrm{m}$ in diameter with a two layers $62.5 \mu \mathrm{m}$ thick epoxy-acrylate polymer coating. Both as-received optical fiber and stripped fiber were subjected to the uniaxial tensile test and the cantilever beam bending test. The stripped fiber Young's modulus results under both tests were found to be very similar. Thus, the mark-tracking method is adaptable to the tensile test of optical fibers and the elastic behavior of both as-received optical fiber and stripped fiber is found to be linear. Their Young's modulus are $22 \mathrm{GPa}$ and $83 \mathrm{GPa}$, respectively. These results revealed that those coatings are playing a mechanical role in the fiber elongation.
\end{abstract}

\section{Introduction}

Besides signal transmission for telecommunications, fibers are used in an increasing number of devices and the availability of silica fibers enlarges the field of the possible applications such as optical fiber sensors, remote chemical analysis, thermal measurements and thermal imaging, reflectometry, optical instrumentation and also laser power delivery [1-2]. Most medical applications of fibers, with their specific requirements, are related to this group. Moreover, optical fibers sensors are widely used in smart materials [3-5], for example in order to monitor civil engineering structures to assess their durability. In the framework of this study, we use composite material embedded optical fibers in order to improve the use in service of High Technology Bridge. Therefore, elastic properties have been a major consideration in the application of optical fibers. In order to characterize the elastic properties of optical fiber, both as-received fiber and stripped fiber (stripped polymer coating fiber) were subjected to mechanical tests. In this work, cantilever beam bending test and uniaxial tensile test were performed to determine the Young's modulus of optical fiber. Optical fibers used in this study are commercial Verrillon single mode silica fibers which are $125 \mu \mathrm{m}$ in diameter with a two layers $62.5 \mu \mathrm{m}$ thick epoxy-acrylate polymer coating. This type of coating is the most widely used in standard optical fiber.

This is an Open Access article distributed under the terms of the Creative Commons Attribution-Noncommercial License 3.0, which permits unrestricted use, distribution, and reproduction in any noncommercial medium, provided the original work is properly cited. 
The Young's modulus of stripped fiber is determined by a cantilever beam test. Then, to validate this result some uniaxial tensile tests were performed. The uniaxial tensile test was usually used to characterize the mechanical behavior of optical fiber by many studies [6-7]. In this paper, we also used this kind of test by using an optical method (mark-tracking method) to measure fiber elongation instead of using the crosshead machine displacement.

\section{Experimental Tests}

\subsection{Cantilever beam bending test}

A cantilever is a beam supported only on one end as shown on figure $1 \mathrm{~b}$. This test, thanks to strength of materials theory, leads to determine the Young's modulus of silica fiber (stripped polymer coated fiber) [8]. The gauge length of the specimen is $244 \mathrm{~mm}$ and stripped fiber density $\rho$ is $2.15 \mathrm{~g} / \mathrm{cm}^{3}$ (measured by a helium pycnometry).
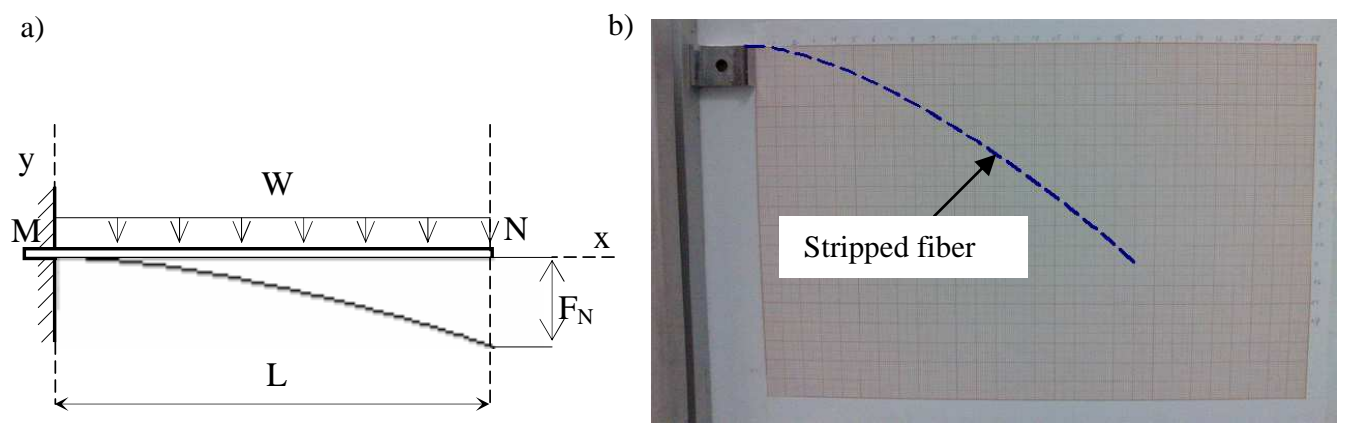

Fig. 1: Cantilever beam test; a) schema of cantilever beam b) cantilever beam test of stripped fiber

The geometry of the bended fiber and the coordinate system that will be used is on figure 1a.

Deflection along the beam is given by:

$$
\begin{aligned}
& y=\frac{-W x^{2}}{24 E I}\left(x^{2}-4 L x+6 L^{2}\right) \\
& I=\frac{\pi d^{4}}{64} \\
& W=\rho \cdot g \cdot \frac{\pi \cdot d^{2}}{4}
\end{aligned}
$$

Where: E and I are respectively the Young's modulus and the moment of inertia of the stripped fiber

$\mathrm{W}$ is the self weight load $(\mathrm{N} / \mathrm{mm})$

$\mathrm{d}$ is the diameter of the stripped fiber and is $125 \mu \mathrm{m}$ measured by optical microscopy and also SEM (Scanning Electronic Microscope).

$\mathrm{g}$ is equal to $9.81 \mathrm{~m} / \mathrm{s}^{2}$ 
With deflection measured at the free end of the beam, the Young's modulus of a stripped polymer coating fiber is calculated as:

$$
E=\frac{-q L^{4}}{8 f_{N} I}
$$

Where $\mathrm{f}_{\mathrm{B}}$ is elastic deflection in $(\mathrm{mm})$ at point $\mathrm{N}$.

The diameter of the core (wave guide) doped by Germanium is $8 \pm 1 \mu \mathrm{m}$ and is positioned on the neutral axis of the beam so that the Young's modulus obtained is the one of silica. The deflection measured at the end of the beam $f_{N}$ is $114 \mathrm{~mm}$ corresponding to a Young's modulus of stripped polymer coating fiber $\mathrm{E}=83 \mathrm{GPa}$, which is different from the one of silica fiber $\mathrm{E}=73 \mathrm{GPa}$ found in the literature [6-7]. Therefore, to confirm this value, a uniaxial tensile test with the attachment of mark tracking method was performed as follow.

\subsection{Uniaxial tensile test with mark-tracking method}

\subsubsection{Mark-Tracking method}

The mark-tracking method is the optical technique allowed for local strain measurement [9]. For this study, two markers are deposited, gently and carefully in order to avoid the incidental surface cracks, on the surface of specimens with permanent marker pen. Determination of strain was done under the principle of measurement mentioned above.

\subsubsection{Automatic measurement devices}

Algorithm of the image processing allows us to calculate the barycenter coordinates of the two markers applying on the sample.

The coordinates of the marks are given by the following equations [10]:

$$
\left\{\begin{array}{r}
x_{g}=\frac{\sum_{i} x_{i}\left(I_{i}-I_{s}\right)}{\sum_{i}\left(I_{i}-I_{s}\right)} \\
y_{g}=\frac{\sum_{i} y_{i}\left(I_{i}-I_{s}\right)}{\sum_{i}\left(I_{i}-I_{s}\right)}
\end{array}\right.
$$

Where $\mathrm{I}_{\mathrm{i}}$ is the grey level of the pixel whose coordinates are $\left(x_{i}, y_{i}\right)$ and $\mathrm{I}_{\mathrm{s}}$ is the threshold value by which the pixels of markers are distinguished.

As this method is operated only in reference $(O, \vec{X}, \vec{Y})$, out of plan deformation was controlled carefully by using a mirror inclined $45^{\circ}$ comparing to the axis of Camera CCD. Once the marks are located, the lengths between the two markers $l(t)$ and the strain $\mathcal{E}(t)$ by the time (every second) are calculated following the principle of deformation measurement as this method. 


\subsubsection{Principle of deformation measurement}

In figure 2, the line segment $\mathrm{M}_{1} \mathrm{M}_{2}$ considered as segment of two markers at initial state at time $\mathrm{t}=0$ in the reference $(O, \vec{X}, \vec{Y})$ changes into a segment $\mathrm{M}_{3} \mathrm{M}_{4}$ at time $t$ once the load is applied (collected every second).

Initial state (time $\mathrm{t}=0$ )

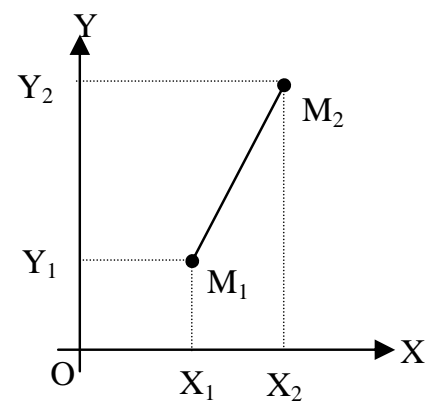

Deformed state (time $\mathrm{t}$ )

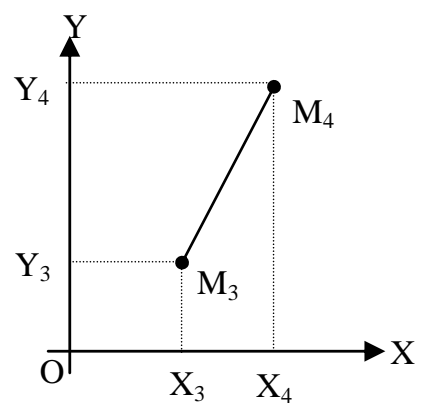

Fig. 2: Deformation of line segment

So the deformed length $l(t)$ and the original length $L$ are given by:

$$
l(t)=\sqrt{\left(X_{4}-X_{3}\right)^{2}+\left(Y_{4}-Y_{3}\right)^{2}}
$$

and

$$
L=\sqrt{\left(X_{2}-X_{1}\right)^{2}+\left(Y_{2}-Y_{1}\right)^{2}}
$$

Strain calculations were performed with respect to the small strains assumption (strain $\leq 1 \%$ ) or the finite strains (strain $>1 \%$ ).

The engineering normal strain or engineering extensional strain $e$ of the fiber axially loaded is expressed as a change in the length $\Delta L$ per unit of the original length $L$ of the line element or fiber. The normal strain is positive if the material fibers are stretched. Thus, we have:

$$
e=\frac{\Delta l}{L}=\frac{l(t)-L}{L}=\frac{l(t)}{L}-1=\lambda(t)-1
$$

Where $\lambda$ is a stretch ratio or extension ratio and it is defined as the ratio between the final length $\ell(t)$ and the initial fibers length $L$.

Typical normal strain, $e$, is greater than $1 \%$, thus we use the logarithm strain or true strain in this study.

The logarithm strain considered as an incremental strain is given by:

$$
\delta \varepsilon=\frac{\delta l}{L}
$$


and the logarithmic strain is obtained by integrating this incremental strain:

$$
\begin{aligned}
& \int \delta \varepsilon=\int_{L}^{l(t)} \frac{\delta l}{L} \\
& \varepsilon(t)=\lg \left(\frac{l(t)}{L}\right)=\lg (\lambda(t))
\end{aligned}
$$

The parameters which are to be determined are the coordinates of $\mathrm{M}_{1}, \mathrm{M}_{2}$ (Initial state) and $\mathrm{M}_{3}, \mathrm{M}_{4}$ (deformed state). Then we can calculate the axial strains of the fiber, which can be small $(\leq 1 \%)$ or large $(>1 \%)$.

\subsubsection{Tensile Tests}

The monitor was used to control the fiber deformation during the test (spot diffraction or markers images). Recording of markers positions is done with a CCD video camera sensor and a data acquisition card. Then all data information was stored in a computer for strain calculations, with corresponding tensile forces data for stress calculations.

a)

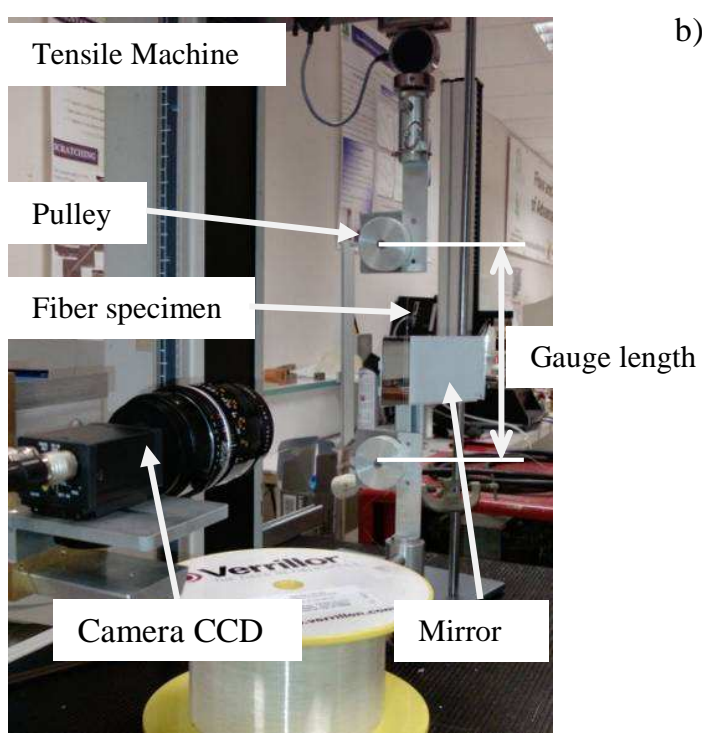

Fig. 3: a) Data acquisition system devices b)

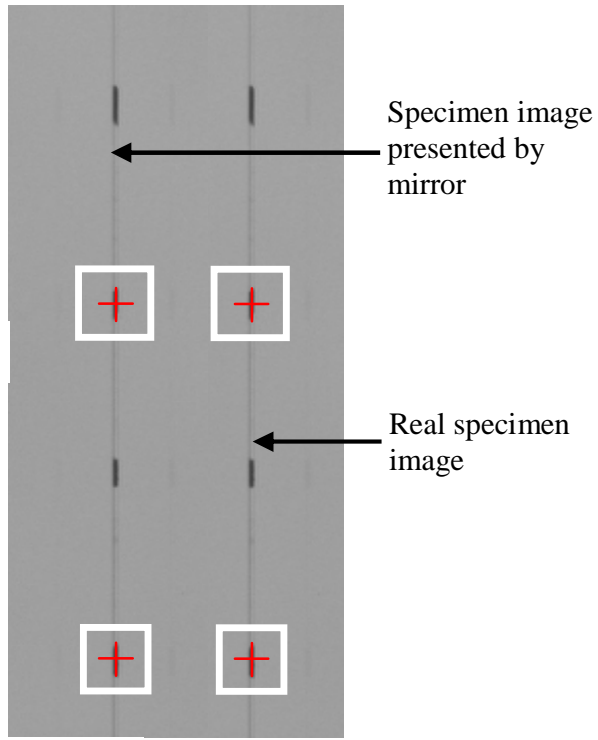

b) Image analysis

Optical fibers were wind round the pulleys to perform the tensile test as shown on figure 3 . The gauge length was $0.2 \mathrm{~m}$ and the tests were performed at room temperature $\left(19 \pm 1^{\circ} \mathrm{C}, 40 \sim 50 \% \mathrm{RH}\right)$. The cross-head speeds were $8 \mathrm{~mm} / \mathrm{mn}$ and $4 \mathrm{~mm} / \mathrm{mn}$ for polymer coated fiber and stripped polymer coating fiber respectively. In order to avoid fibers slipping and damages on the contact area double sided tap was applied on the literal surface of each pulley on which tree windings of fiber specimen were carried out. For the stripped polymer coating fiber specimen, stripped length was $2 \mathrm{~cm} \sim 3 \mathrm{~cm}$ in the middle part of the specimen (polymer coated fiber). The tensile strength of the optical fiber $\sigma$ is calculated by: 


$$
\sigma=\frac{F}{A}
$$

Where: $\mathrm{F}$ is the measured force

A is the cross-sectional area of the optical fiber for polymer coated fiber testing or crosssectional area of glass fiber for stripped polymer coating fiber.

\section{Experimental results and discussion}

The main purposes of these tensile tests are to find out Young's modulus of polymer coated fiber (PCF) and stripped fiber (SF), and also its linearity.

During the tensile tests, none of any out of plan deformation were noticed as the strain curves of the real specimen and of the mirror-presented specimen were superimposed. As a result, the marktracking method was available. Table1 and table2 revealed that Young's modulus of as-received fiber and stripped fiber were found to be $22 \mathrm{GPa}$ and $83 \mathrm{GPa}$ with an elastic linear behavior in term of deformation $5 \sim 6 \%$ and $3 \%$ respectively as shown on figure 4 .

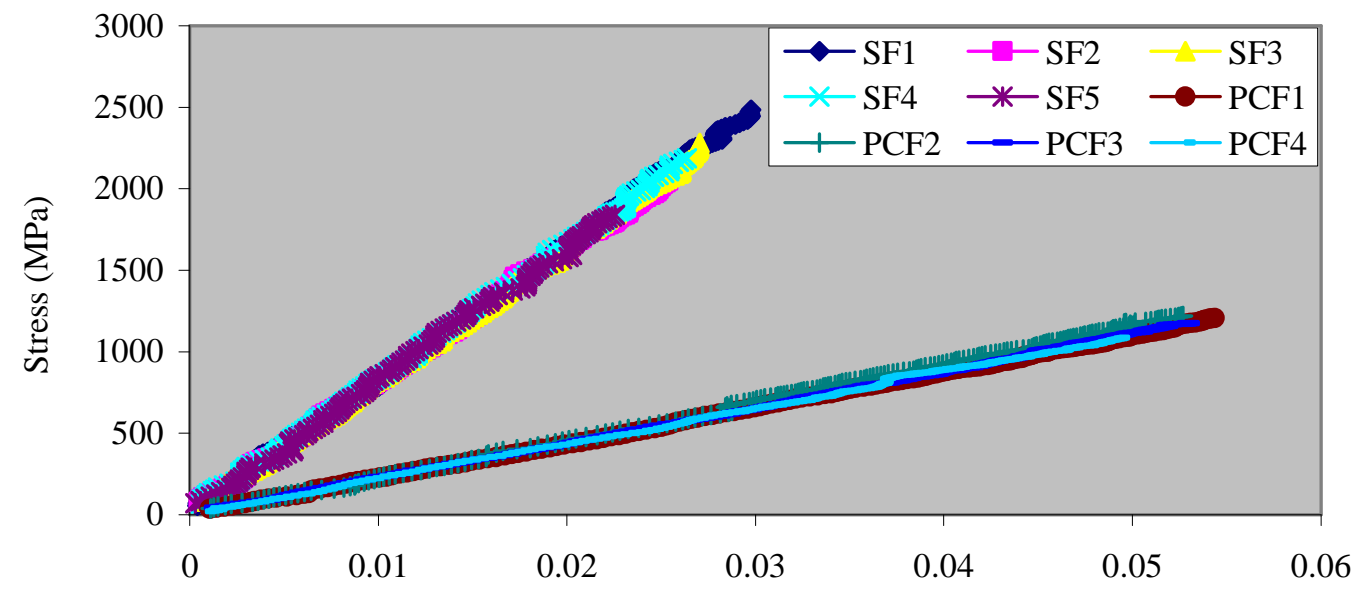

Strain

Fig. 4: Stress-strain curve of polymer coated fiber (PCF) and stripped fiber (SF)

Their mechanical properties were reported and given by table1 and table2.

Table 1: Mechanical property of as-received fiber

\begin{tabular}{cccc}
\hline $\mathrm{N}^{\circ}$ & Diameter $\times 10^{-3} \mathrm{~mm}$ & Failure strain $(\%)$ & Young's modulus $(\mathrm{GPa})$ \\
\hline 1 & 245 & 5.4 & 21.96 \\
2 & 245 & 5.3 & 23.13 \\
3 & 245 & 5.3 & 22.11 \\
4 & 245 & 5.0 & 22.02 \\
\hline \multirow{3}{*}{} & Average & 22.30 \\
& & Standard deviation & 0.55 \\
\cline { 3 - 3 } & &
\end{tabular}


Table 2: Mechanical property of stripped fiber

\begin{tabular}{cccc}
\hline $\mathrm{N}^{\circ}$ & Diameter $\times 10^{-3} \mathrm{~mm}$ & Failure strain $(\%)$ & Young's modulus(GPa) \\
\hline 1 & 125 & 2.3 & 81.92 \\
2 & 125 & 2.5 & 81.12 \\
3 & 125 & 2.7 & 81.91 \\
4 & 125 & 2.2 & 83.38 \\
5 & 125 & 3.0 & 83.42 \\
\hline \multirow{3}{*}{} & Average & 82.35 \\
& & Standard deviation & 1.01 \\
\cline { 3 - 3 }
\end{tabular}

In previous studies, Young's modulus of silica fibers were found to be around 73GPa, which is absolutely different from the results mentioned in our study. Moreover, the stress-strain relation of optical fibers was first examined by Mallinder and Proctor [11] and later by Glaesemann et al. [12]. They found that the relation between stress and strain of an optical fiber is nonlinear. Then, the nonlinear behavior was used in the strength measurement of optical fiber by many studies [13-15]. Hence, there are two aspects to be considered and to be discussed.

The first aspect refers to strain calculation. We would compared the logarithm strain in our case, with the small assumption strain, e, and also with the crosshead displacement-characterized strain. As the strains results varied from $3 \%$ to $6 \%$, the strains calculated by the small assumption, e, and the logarithm ones are very similar. Once using the crosshead displacement-characterized strains, figure 5 shown that the mechanical behavior of stripped fiber was nonlinear, but the Young's modulus value is about only 40GPa which was two times smaller than the one measured by using the mark-tracking method.

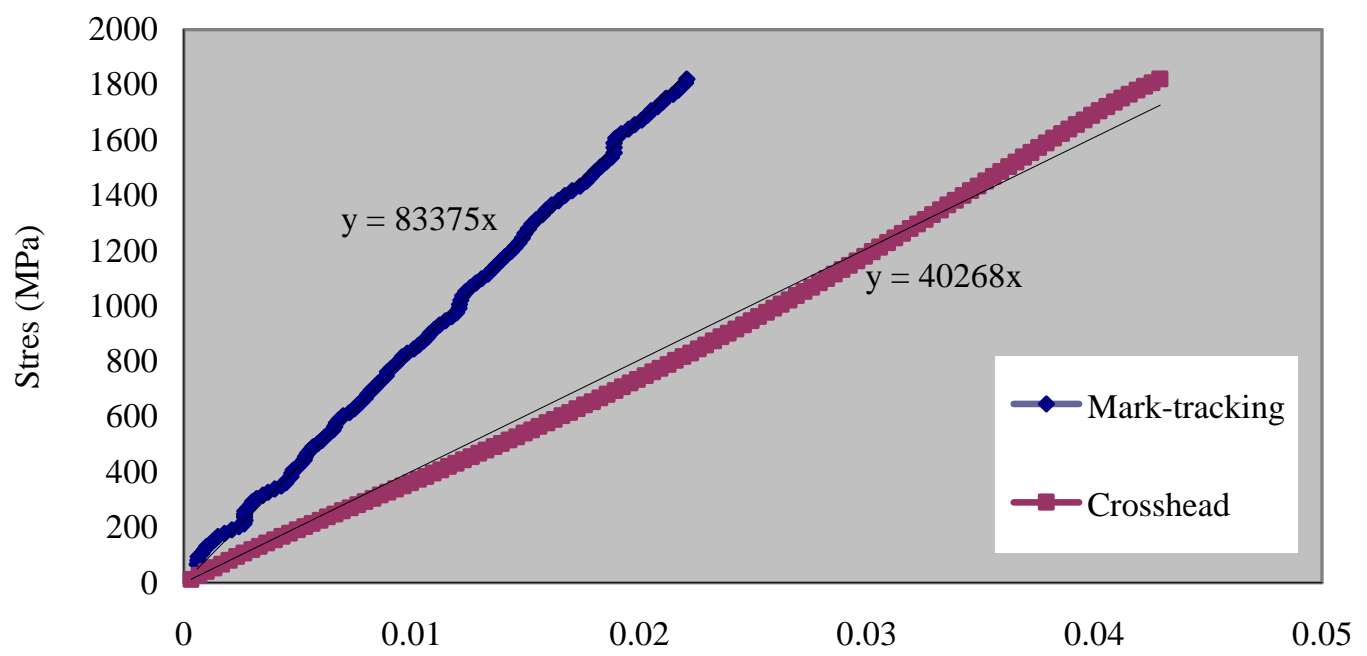

Strain

Fig. 5: Stress-strain curves of stripped fiber specimen $n^{\circ} 4$

The second aspect refers to the Young's modulus aspect. We found that Young's modulus of the stripped fiber is $83 \mathrm{GPa}$ which seems to be a high value compared to the one of fused silica fiber 
usually about $73 \mathrm{GPa}$ whose behavior was nonlinear. Optical fibers used for this study are commercial Verrillon single mode silica fibers which may be different from the other commercial fibers. Thus, this high value of the Young's modulus and the linear relation between stress and strain might be caused by the concentration of fluoride in this optical fiber cladding. Consequently, we will do some additional tests to observe the linearity of the behavior on other standard commercial optical fibers whose Young's modulus is 73GPa.

On the part of as-received fibers, the fibers were considered as a composite material whose Young's modulus could be calculated by respecting the mixture model of Voigt. This Young's modulus was finally confirmed by experimental tests in this study.

\section{Conclusion and perspective}

The cantilever beam bending test is an effective test allowing a rapid measurement of the Young's modulus of the fiber. The tensile test provides not only the Young's modulus, but also its linearity presented by the stress-strain curves. With the attachment of the mark-tracking method in the tensile test, we proved that the Young's modulus of the as-received fiber and the stripped one are differently about $22 \mathrm{GPa}$ and $83 \mathrm{GPa}$ with an elastic linear behavior in term of deformation $5 \sim 6 \%$ and $3 \%$ respectively. The Young's modulus of the stripped fiber is the same using cantilever beam bending test or uniaxial tensile test. These results point out those coatings playing a mechanical role in the fiber elongation. Thus, the mark-tracking method is finally the potential and available technique for optical fiber mechanical properties measurements.

According to the authors, other kind of commercial fibers will be tested with the same experimental protocol test in order to study the linear or nonlinear behavior in the stress-strain relation.

\section{References}

1. I.B. Kwon, M.Y. Choi, H. Moon, Sensors and Actuators A 112, 8 (2004)

2. B. Lee, Optical Fiber Technology 9, 23 (2003)

3. M.V. Gandhi, B.S. Thompson, Smart Materials And Structure, CHAPMAN \& HALL, 309 (1992)

4. C.S. Shin, C.C. Chiang, Internation Journal of Fatigue 28, 7 (2006)

5. M. Majumder, T.K. Gangopadhyay, A.K. Chakraborty, Sensors and Actuators A 147, 15 (2008)

6. B. Delobelle, F. Thiebaud, D. Chappelle, D. Perreux, V. Placet, R. Ferrière, Comptes Rendus des JNC 16-Toulouse, 10 (2009)

7. C.P. Chen, T.H. Chang, Materials Chemistry and Physics 77, 7 (2002)

8. J.L. Fanchon, Guide de MECANIQUE science et technologies industrielles, Editions Natan, 480 (1996)

9. F. Brémand, J.-C. Dupré, Lagarde, Proc. Photomécanique 95 - Etude du comportement des matériaux et des structures, 7 (1995)

10. R. Rotinat, R. Tié bi,V. Valle, J.-C. Dupré, Strain No.3 Vol.37, 10 (2001)

11. E.P. Mallinder, B.A. Proctor, Physis and chemistry of glass 5, 13 (1964)

12. G.S. Glaesemann, S. T. Gulati, J. D. Helfinstine, Tech.Dig. $-11^{\text {th }}$ opt. Fiber Comm. conf., TUG5, (1988)

13. W. Griffioen, J. Am. Ceram. Soc., 75, 5 (1992)

14. M. John Matthewson, Proceeding of a conference held 8-9 Septemberboston, Massahusettes, 28 (1994)

15. Prabhat K. Gupta, Charles R. Kurkjian, Journal of Non-Crystalline Solids 351, 5 (2005) 